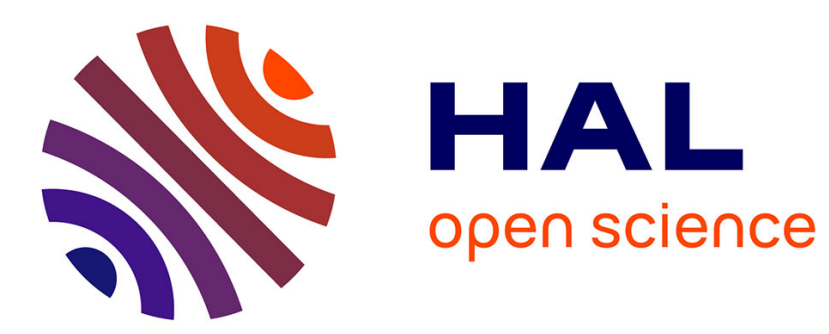

\title{
A Gait Analysis Method Based on a Depth Camera for Fall Prevention
}

\author{
Amandine Dubois, François Charpillet
}

\section{To cite this version:}

Amandine Dubois, François Charpillet. A Gait Analysis Method Based on a Depth Camera for Fall Prevention. The 37th Annual International Conference of the IEEE Engineering in Medicine and Biology Society (EMBS), Aug 2014, Chicago, United States. hal-01069640

\section{HAL Id: hal-01069640 \\ https://inria.hal.science/hal-01069640}

Submitted on 29 Sep 2014

HAL is a multi-disciplinary open access archive for the deposit and dissemination of scientific research documents, whether they are published or not. The documents may come from teaching and research institutions in France or abroad, or from public or private research centers.
L'archive ouverte pluridisciplinaire HAL, est destinée au dépôt et à la diffusion de documents scientifiques de niveau recherche, publiés ou non, émanant des établissements d'enseignement et de recherche français ou étrangers, des laboratoires publics ou privés. 


\title{
A Gait Analysis Method Based on a Depth Camera for Fall Prevention
}

\author{
Amandine Dubois ${ }^{1,2,3}$ and Francois Charpillet ${ }^{1,2,3}$
}

\begin{abstract}
This paper proposes a markerless system whose purpose is to help preventing falls of elderly people at home. To track human movements, the Microsoft Kinect camera is used which allows to acquire at the same time a RGB image and a depth image. Several articles show that the analysis of some gait parameters could allow fall risk assessment. We developed a system which extracts three gait parameters (the length and the duration of steps and the speed of the gait) by tracking the center of mass of the person. To check the validity of our system, the accuracy of the gait parameters obtained with the camera is evaluated. In an experiment, eleven subjects walked on an actimetric carpet, perpendicularly to the camera which filmed the scene. The three gait parameters obtained by the carpet are compared with those of the camera. In this study, four situations were tested to evaluate the robustness of our model. The subjects walked normally, making small steps, wearing a skirt and in front of the camera. The results showed that the system is accurate when there is one camera fixed perpendicularly. Thus we believe that the presented method is accurate enough to be used in real fall prevention applications.
\end{abstract}

\section{INTRODUCTION}

The ageing of the population is a major problem for the next years. One of the problems generating a loss of autonomy is fall. Each year a third of the persons aged 65 years and older fall [3]. Falls have multiple risk factors: age, muscular weakness, gait modification, obstacles in the environment (carpet)... Fall risk can be detected by measuring gait modifications.

Several systems exist for computing gait parameters. There are systems like accelerometers, gyroscopes which measure the angular velocity of the segment on which they are put. There exist optical systems (as the VICON system) for movement tracking based on markers fixed on certain key points of the body. Other systems without wearable sensors exist as for example force platforms, actimetric carpets, on which the person walks and which detect the exact moment of the foot-ground contact and calculate the velocity and the length of steps. There are also telemeters computing velocity. We would like to develop a system based on a low cost and not imposing ambiant sensor (without wearable markers). In the litterature, some authors begin to be interessed in developing ambiant systems allowing to analyze the gait of a person. In 2009 Hagler et al [5] installed four PIR motion sensors on the ceiling to obtain the speed of the gait. Auvinet et al [2] in 2011 are interessed in detecting symmetrical gait troubles with three RGB-D cameras. In 2011 Stone et

\footnotetext{
${ }^{1}$ Inria, Villers-lès-Nancy, 54600, France.

${ }^{2}$ Université de Lorraine, LORIA, UMR 7503, Vandoeuvre-lès-Nancy, 54506, France.

${ }^{3}$ CNRS, LORIA, UMR 7503, Vandoeuvre-lès-Nancy, 54506, France.
}

al [9] developped a system to evaluate a fall risk with an inexpensive depth camera. The idea is to measure some gait parameters of the person with a RGB-D camera.

The aim of our system is to record and to analyze the gait parameters of a person regularly enough to note a possible evolution over time. On some period, the aim is to know if the gait parameters tend to remain stable or if they are evolving which would suggest an increased risk of fall. In the last case, the results could be transmited to the physician.

The proposed system uses a RGB-D camera chosen for the advantage of the depth reconstruction, its low cost and its invariance to visible lighting. We imagine to fix the system high in a corner at home. Several stages are necessary to achieve our goal. The system must be able to detect the person in the field of view, to detect when she walks and finally to analyze her gait. The algorithm that we use to extract the person and to detect her activity (as walking) is presented in [4]. In this article, we present an algorithm for analysing the gait. Our method consists in extracting gait parameters, of interest for fall prevention, from the trajectory of the center of mass.

This paper is organized as follows. Section II presents background elements on activity recognition that we use for detecting walking situations. Section III presents the algorithm allowing to analyze the gait of a person. Section IV presents the evaluation of our algorithm.

\section{BACKGROUND}

This work is the continuation of previous work on fall detection with RGB-D camera [4]. The detection of the falls was included in the more general problem of activity recognition. The method consists in tracking a person in the field of the view of the camera. To extract the person from the background, a standard method in computer vision is used. A Hidden Markov Model (HMM) is used to analyze the activity of the person. Each state represents an activity that the person can do in her daily life. Eight activities were defined: walking, lying (on a bed, on a couch for example), sitting, falling, lying down, squatting, going up on an obstacle (a chair, a footboard for example) and bending. These activities are distinguished using 3 parameters: the position of the center of mass of a person on vertical axis, the vertical speed and standard deviation of the person's body. To know in what state the person is, the Forward-Backward algorithm is used. 26 healthy subjects, of 20 at 53 years old, performed the 8 activities, which represented a training database of 208 sequences. The observation probabilities of the three parameters for each of the 8 situations were learnt from of 128 sequences. The validity of the algorithm was 
been tested with 80 sequences. The results showed that the algorithm could distinguished the different activities. The only error was for the state "bending" which was replaced by state "sitting" or "squatting".

The HMM algorithm provides the state of the person and the general idea is to analyze the gait of the person when she is in the state "walking". The following stage consists in analyzing the gait.

\section{METHOD}

The background extraction is realized using the "running average" method as presented in [4]. In this part, the method for tracking the person and analyzing the gait is presented.

\section{A. Tracking of the person}

To obtain the displacement of the walking body, our approach consists in analyzing the displacement of her center of mass. The horizontal and vertical displacements of the center of mass when a person walks is described in Figure 1(a) and 1(b).

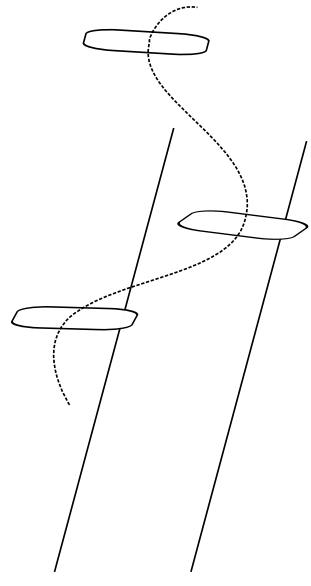

(a) Horizontal displacement.

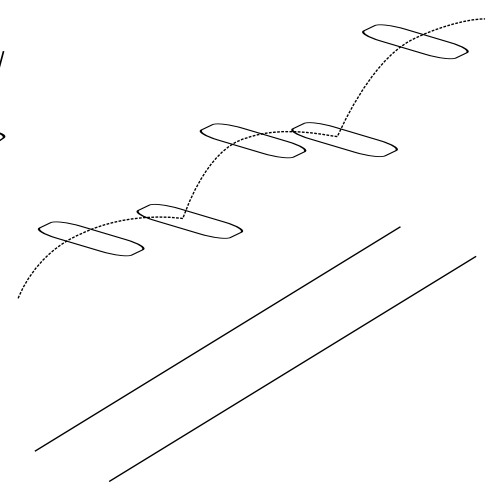

(b) Vertical displacement.
Fig. 1. Displacement of the center of mass as described in the literature [8].

The background extraction allows us to have the mobile pixels belonging to the person. To calculate the center of mass of the person, the method consists in averaging the location of all the mobile pixels of the person. The displacement of the center of mass of a person walking perpendicular to the camera, obtained with our method, is drawn in Figure 2. The horizontal trajectory of the Figure 2(b) is smoother than the trajectory described in litterature. The vertical trajectory of the Figure 2(a) is similar to the theoretical sinusoidal curve described by Saunders in Figure 1(b). Only the displacement on the vertical axis is discussed in the following section. The center of mass trajectory is smoothed with a Kalman filter [7].

\section{B. Analysis of the gait}

1) Selection of gait parameters: To analyze the gait of a person, some parameters can be cited according the purpose of the analysis. A first category corresponds to the spatiotemporal parameters, as the length of steps, the duration

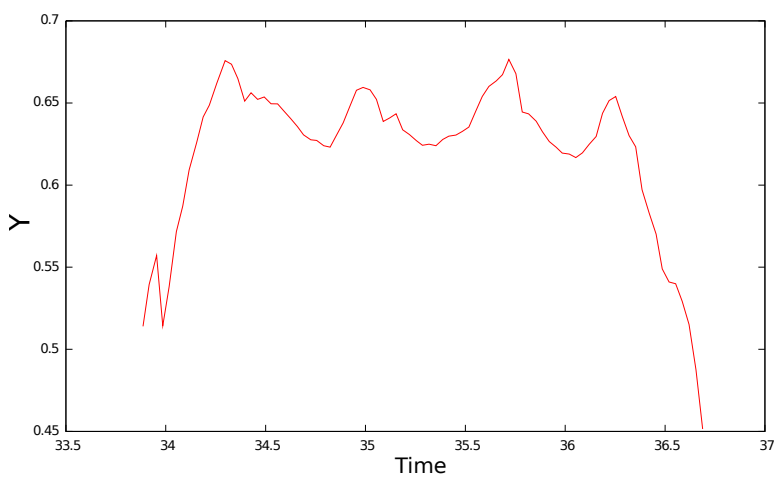

(a) Vertical displacement.

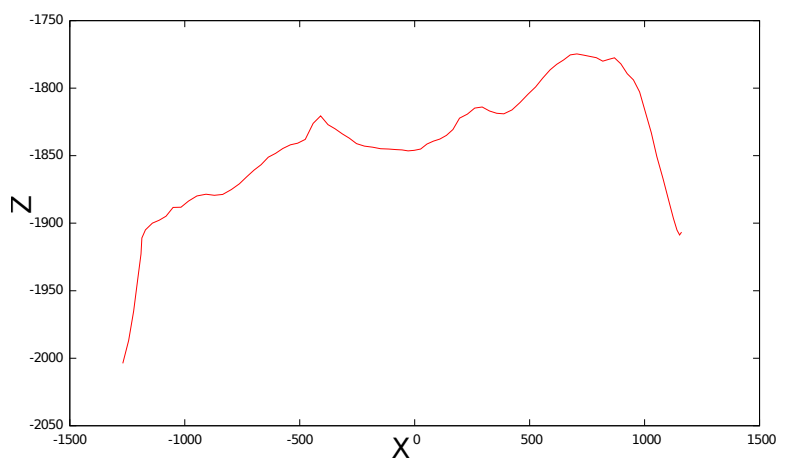

(b) Horizontal displacement.

Fig. 2. Displacement of the center of mass extracted by our algorithm (before filtering) for a person walking in straight, perpendicularly of the camera.

of steps, the speed of the gait, the time propulsion, which are widely studied because they illustrate the characteristics of the gait. Then, the kinematic parameters, as knee angle, are the indicators reflecting the movement of the lower limbs during walking. And we can also cite the dynamic parameters, as the reaction forces from the ground and the center of pressure, allowing an evaluation of the foot.

Our aim is to develop a system for fall prevention through a global gait analysis, so the first category (the spatiotemporal parameters) proves to be more suitable. Several articles show that some gait parameters are indicators of an increased fall risk. Hausdorff et al [6], in 2001, on a one year experiment showed that the stride time variability, the swing time variability and the inconsistency of the variance is associated with an increased risk of future fall. Logistic regression also showed that stride time variability predicts falls. Auvinet et al [1] showed in 2003 that the speed, the frequency and the length of the steps had reduced values for fallers. The irregularity of the length of steps is considered by them as a reliable variable to predict falls. We are interested in the gait parameters as indicators for the prevention of falls. Therefore we focus, according to the litterature, on measuring the length and duration of the steps and the speed of the gait

2) Extraction of the selected gait parameters: The gait parameters (length and duration of steps and the speed of the gait) are extracted from the vertical displacement of the center of mass. In medicine the definition of the length of 
steps is the distance separating the two heels at the time of double support, as illustrated in Figure 4. Our method consists in searching the position of the supporting foot. The center of mass is located at the vertical of the supporting foot when it is highest as shown in Figure 3. The position of the supporting foot is estimated by the projection on the ground of the center of mass (at the time of the maxima). The length of step is calculated as the distance between the projection of two local vertical maxima of the trajectory of the center of mass. In the same way the duration of the steps, representing the time separating two consecutive double support instants, is calculated as the time between the projection of two consecutive local vertical maxima of the trajectory of the center of mass. The speed of the gait is calculated, on a straight line, between the moment of appearance of the person in the field of view of the camera and its last moment of appearance. The extraction of the three gait parameters is shown in Figure 3.

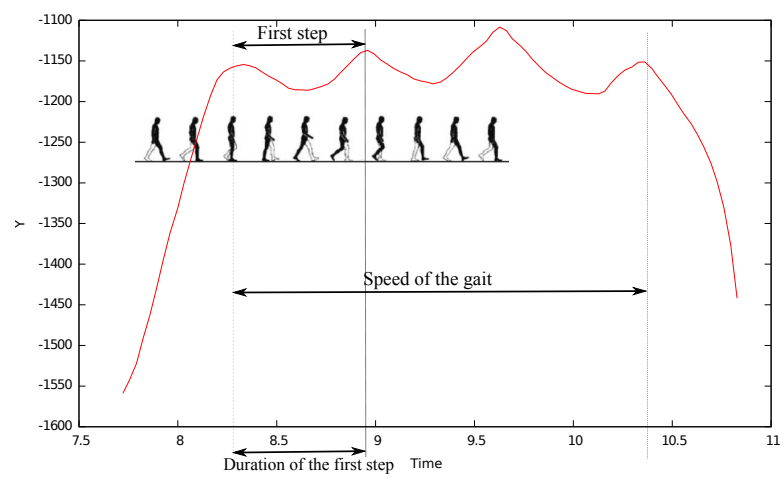

Fig. 3. Extraction of the gait parameters from the center of mass.

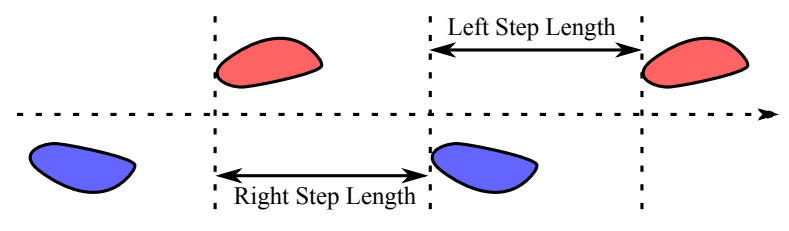

Fig. 4. Definition of the step length.

An illustration of the acquisition system is given in Figure 5. The background image is represented at the top. The vertical trajectory of the center of mass is drawn in the bottom right-hand corner with the measured step lengths.

\section{Evaluation OF THE ALGORITHM}

\section{A. Description of the experiment}

Eleven subjects took part in the experiment among which four women and seven men aged from 22 to 53. The experiment consisted in using an actimetric carpet (GAITRite) of 5 meters length, as a reference for evaluating our system. The error of measurement is $1.27 \mathrm{~cm}$ which is the distance between the sensors in the carpet. The experiment was held in a smart room where the sujects were filmed by a camera RGB-D. The field of view of the camera is $3 \mathrm{~m}$ wide and $6 \mathrm{~m}$ deep. The persons walked perpendiculary to the camera

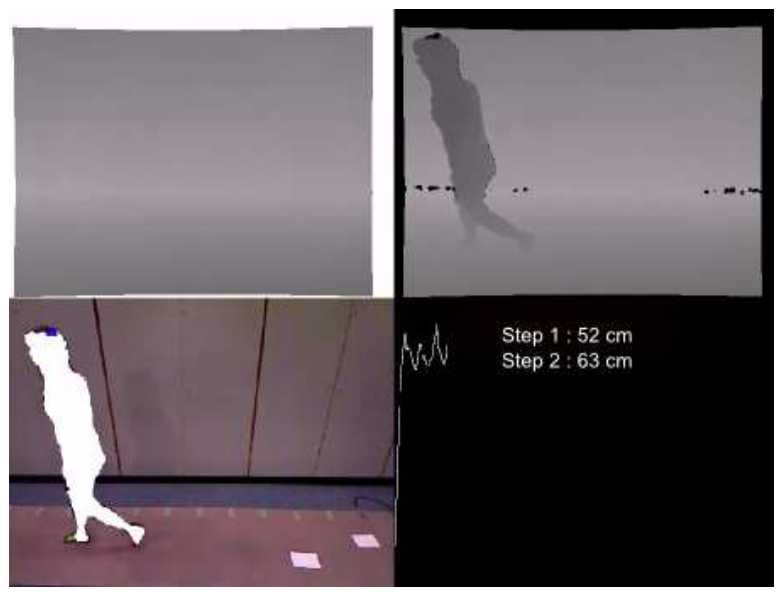

Fig. 5. Gait acquisition screen capture.

(except for a situation that we describe in the following paragraph), at a distance of $3 \mathrm{~m}$ from the camera.

The instruction given to the subjects was to make 4 situations. Each subject had to walk three times in every situation to have a significant amount of data (165 sequences were analysed). The situations were to:

- walk in a straight line "normally";

- walk straight making small steps;

- walk with a long skirt to know if the clothes disturb the gait (for all the subjects);

- walk in front of the camera (the other situations are performed with the camera perpendicular at the subject).

The gait parameters obtained with the carpet were compared to the values provided by the camera algorithm. In this experiment we compared three gait parameters: length and duration of the steps and the speed of the gait.

\section{B. Results}

1) Results based on the error on each sequence: The mean of the length, the duration of the steps and the speed of gait are calculated for the carpet and our algorithm for each sequence. A sequence is a walk composed of 2 to 5 steps, depending on the subject and the situation. The error between the mean obtained with the carpet and the camera is calculated. Then the average error is obtained for each situation. The results are shown in Table I.

For the length of steps the average error for situations "Normal", "Small" and "Skirt" (all exept "Front") is less than 4.7\%.

For the duration of steps, the average error for all situations (exept "Front") is less than 6.6\%.

For the speed of the gait, the average error for situation "Normal" and "Small" is less than 3.69\%. For the "Skirt" situation, the average error is more important, $4.37 \%$. The situation with the camera in front of the subject is the worst for the accuracy of the speed.

Only the error on the situation with the camera in front is important. When the camera is in front of the subject the results are degraded. The system gives accurate enough 
values on all the other situations. The speed of gait is the most accurate of the gait parameters.

\begin{tabular}{|c|c|c|c|c|} 
& Normal & Small & Skirt & Front \\
\hline Length & $4.6 \mid 3.8$ & $4.7 \mid 4.2$ & $4.7 \mid 3.3$ & $12.5 \mid 12.6$ \\
\hline Duration & 6.614 .9 & $6.1 \mid 4.5$ & $4.7 \mid 5.5$ & $11.5 \mid 8.3$ \\
\hline Speed & $3.69 \mid 1.81$ & $3.28 \mid 2.38$ & $4.37 \mid 3.19$ & $10.04 \mid 7.33$ \\
\hline
\end{tabular}

TABLE I

$\%$ DifFERENCE AND STANDARD DEVIATION OBTAINED ON SEQUENCE AVERAGE OF THE PARAMETERS FOR THE 4 SITUATIONS

(AVERAgelStD.DEViation).

2) Results based on the mean of each gait parameter: Another way to analyze the results is to calculate the mean of each parameter of the gait. The results are shown in Table II. In this table the mean for the three gait parameters is calculated for the actimetric carpet and for our camera system.

First for the steps length, we can see that the mean obtained with the carpet and with the camera are similar for all the situations. The maximum error between carpet and camera is $2 \mathrm{~cm}$. The situation with the small steps is as accurate as the normal steps.

For the duration of the steps, the mean maximum error is $0.04 \mathrm{~s}$ for all the situations.

For the speed of the gait, the error is more important in the "Front" situation than in the other situations. In the "Front" situation, the mean error is $6.3 \mathrm{~cm} / \mathrm{s}$. In the other situations, the mean error is less than $3.7 \mathrm{~cm} / \mathrm{s}$.

In this part, we can see that our system provided values similar to those provided by the carpet in all the situations when we are interested in the mean of the length and duration of the steps and in the speed of the gait.

\begin{tabular}{|c|c|c|c|c|}
\hline & & Length $(\mathrm{cm})$ & Duration(s) & Speed(cm/s) \\
\hline Sit & System & Mean & Mean & Mean \\
\hline 1 & Carpet & 67.6 & 0.66 & 108.7 \\
\hline & Camera & 67.0 & 0.63 & 112.4 \\
\hline 2 & Carpet & 38.4 & 0.71 & 56.9 \\
\hline & Camera & 38.3 & 0.68 & 58.6 \\
\hline 3 & Carpet & 57.1 & 0.68 & 92.0 \\
\hline & Camera & 59.3 & 0.67 & 95.7 \\
\hline 4 & Carpet & 67.2 & 0.66 & 108.8 \\
\hline & Camera & 65.4 & 0.63 & 102.5 \\
\hline
\end{tabular}

TABLE II

ACCURACY OF THE MEASURED GAIT PARAMETERS COMPARED TO THE ACTIMETRIC CARPET (1: NORMAL STEPS, 2: SMALL STEPS, 3: SITUATION WITH SKIRT, 4: FRONT CAMERA).

\section{CONCLUSION}

The aim of our work is to develop a system which allow fall risk assessment from the quality of the person gait. From the camera RGB-D localized at the person home, we track the person and detect when she walks to measure her gait parameters (to detect a possible variation in the quality of the gait). In this paper, the analysis of the gait is presented, knowing that the first stages (tracking of the person and detected that she walks) are already been described in a previous article. The camera provides the depth map that we treat directly. The trajectory of the center of mass is the only information which allows us to track the person and to extract the gait parameters. Three parameters are extracted because they are considered as important to determine a fall risk, the length and duration of steps and the speed of the gait. To evaluate the accuracy of the gait parameters provided by the camera algorithm, we set up an experiment consisting in comparing the measurements provided by the camera to the parameters obtained by an actimetric carpet. The result is that our algorithm provides values for the gait parameters close enough to the reference values when the person walks normally, making small steps and wearing a skirt. When the person walks in front of the camera the results are degraded. This implies that if we fix a camera at home, we must detect the moment where the person is perpendicular to the camera to analyse her gait. We would like to know if our method allows us to detect an evolution, a reduction of the length of steps over a certain time period. Thus for the future we would like to test our model with an elderly people on the long run.

\section{REFERENCES}

[1] B. Auvinet, G. Berrut, C. Touzard, N. Collet, D. Chaleil, and E. Barrey. Gait Abnormalities in Elderly Fallers. Journal of Aging and Physical Activity, pages 40-52, 2003.

[2] E. Auvinet, F. Multon, and J. Meunier. Gait analysis with multiple depth cameras. Conference proceedings : Annual International Conference of the IEEE Engineering in Medicine and Biology Society, Jan. 2011.

[3] H. Bourdessol and S. Pin. Prévention des chutes chez les personnes âgées à domicile. Inpes, France, 2005.

[4] A. Dubois and F. Charpillet. Human Activities Recognition with RGBDepth Camera using HMM. Engineering in Medicine and Biology Society (EMBC), 35th Annual International Conference of the IEEE, 2013.

[5] S. Hagler, D. Austin, T. L. Hayes, J. Kaye, and M. Pavel. Unobtrusive and Ubiquitous In-Home Monitoring: A Methodology for Continuous Assessment of Gait Velocity in Elders. IEEE transactions on biomedical engineering, 57(4):813-20, Apr. 2010.

[6] J. M. Hausdorff, D. A. Rios, and H. K. Edelberg. Gait Variability and Fall Risk in Community-Living Older Adults: A 1-Year Prospective Study. Archives of physical medicine and rehabilitation, 82(8):1050-6, Aug. 2001.

[7] L. R. Rabiner. A tutorial on hidden Markov models and selected applications in speech recognition. In Proceedings of the IEEE, pages 257-286, 1989.

[8] J. B. D. M. Saunders, V. T. Inman, and H. D. Eberhart. The major determinants in normal and pathological gait. The Journal of Bone and Joint Surgery, pages 543-558, July 1953.

[9] E. E. Stone and M. Skubic. Evaluation of an Inexpensive Depth Camera for Passive In-Home Fall Risk Assessment. 5th International Conference on Pervasive Computing Technologies for Healthcare (PervasiveHealth) and Workshops, pages 71-77, 2011. 